\title{
Consistency in End-of-Life Care Preferences Between Hospitalized Elderly Patients and Their Primary Family Caregivers
}

This article was published in the following Dove Press journal: Patient Preference and Adherence

\author{
I-Fei Chuang' \\ Yea-Ing Lotus Shyu iD ${ }^{2-4}$ \\ Li-Chueh Weng (iD) 2,5 \\ Hsiu-Li Huang ${ }^{6}$ \\ 'Department of Nursing, Taichung \\ Veterans General Hospital, Taichung, \\ Taiwan; ${ }^{2} \mathrm{~S} c h o o l$ of Nursing, College of \\ Medicine, Chang Gung University, \\ Taoyuan City, Taiwan; ${ }^{3}$ Healthy Aging \\ Research Center, Chang Gung University, \\ TaoyuanCity, Taiwan; ${ }^{4}$ Traumatological \\ Division, Department of Orthopedics, \\ Chang Gung Memorial Hospital, Taoyuan \\ City, Taiwan; ${ }^{5}$ Department of General \\ Surgery, Chang Gung Memorial Hospital, \\ Taoyuan City, Taiwan; ' Department of \\ Long-TermCare, College of Health \\ Technology, National Taipei University of \\ Nursing and Health Sciences, Taipei, \\ Taiwan
}

Purpose: This study explored the consistency between preferences for end-of-life care for elderly hospitalized patients and their primary caregivers and predictors of consistency.

Patients and Methods: This cross-sectional correlational study recruited 100 dyads of elderly hospitalized patients and their primary caregivers from a medical center in Central Taiwan. A structural questionnaire about preferences for seven end-of-life medical treatment options involved cardiopulmonary resuscitation, intravenous therapy, nasogastric tube feeding, intensive care unit, blood transfusion, tracheotomy, and hemodialysis.

Results: The consistency was $42.28 \%$ for preferences of end-of-life medical care between patients and caregivers. The Kappa values for seven life-sustaining medical treatments ranged from 0.001 to 0.155 . Logistic regression showed that the predictors of consistency for preferences of treatment were: a patient with a signed living will (odds ratio $[\mathrm{OR}]=6.20$, $\mathrm{p}<0.01)$ and a male family caregiver $(\mathrm{OR}=0.23, \mathrm{p}<0.01)$ for cardiopulmonary resuscitation; a patient who visited relatives in the intensive care unit $(\mathrm{OR}=2.94, \mathrm{p}<0.05)$ and a spouse caregiver $(\mathrm{OR}=3.07, \mathrm{p}<0.05)$ for nasogastric tube feeding; a spouse caregiver $(\mathrm{OR}=3.12$, $\mathrm{p}<0.05)$ and a caregiver who visited the intensive care unit $(\mathrm{OR}=5.50, \mathrm{p}<0.01)$ for tracheotomy; and a spouse caregiver $(\mathrm{OR}=2.76, \mathrm{p}<0.05)$ and a caregiver who visited the intensive care unit $(\mathrm{OR}=4.42, \mathrm{p}<0.05)$ for hemodialysis.

Conclusion: End-of-life medical treatment preferences were inconsistent between patients and family caregivers, which might be influenced by Asian culture, the nature of the relationship and individual experiences. Implementation of advance care planning that respects the patient's autonomy and preferences about end-of-life care is recommended.

Keywords: elderly patients, primary family caregivers, end-of-life, care preference, consistency

\section{Introduction}

The population of older adults is growing rapidly and as this population increases, the number of annual deaths also increases, which makes the issue of end-of-life (EoL) decisions more important. Older adults are more likely to have complex comorbidities or life-threatening illnesses that require acute care hospitalization for treatment. If advance directives (ADs) are not in place to describe the patient's preferences for critical or EoL care, hospitals automatically implement lifesustaining medical treatments and cardiopulmonary resuscitation (CPR). ${ }^{1}$ However, for frail older adults, life-sustaining medical treatments and CPR can be ineffective, and unnecessary treatments should be avoided. ${ }^{2}$
Correspondence: Hsiu-Li Huang

No. 83-I, Nei-Chiang St, Wan-Hwa Dist, Taipei 10845, Taiwan

Tel +886-2-28227I 0 I Ext. 6134

Fax +886-2-2389| 464

Email hsiuli@ntunhs.edu.tw 
The capability of older adults to make decisions autonomously often decreases as they age and as the severity of their disease increases. ${ }^{3}$ Consequently, when an older adult has no decision-making capacity, the responsibility to make medical decisions falls on family members, and the family members must make relevant decisions regarding EoL care. A study by Huang et al reported less than half of nursing home residents with dementia in Taiwan had ADs, and most of these had been completed by a family member. ${ }^{4}$ Several studies have demonstrated that there are discrepancies between preferences in EoL care between family members and older adults, ranging from $39.2 \%$ to $71.4 \% .^{5-8}$ Therefore, it is important to ascertain if the preferences of older adults for EoL care are consistent with those of family members. However, most studies on the consistency of EoL preferences have been conducted in Western countries.

Chinese families tend to make decisions as a unit, which differs from decision-making in Western cultures that value individualism. ${ }^{9,10}$ Older adults are at high risk of hospitalization and autonomous decision-making abilities are reduced. However, to date, there is a lack of relevant literature on the consistency between the preferences of older hospitalized patients and their families in Chinese cultures regarding EoL care. Therefore, this study aimed to understand the consistency between the preferences of older adult hospitalized patients and their primary family caregivers regarding EoL life-sustaining medical treatments and relevant factors influencing consistency. The results of this study may provide a reference for the clinical development of ACP. The study could help integrate expectations about EoL care for older hospitalized patients with those of their family caregivers, reduce the decision gap, and provide older hospitalized patients with as comfortable an environment as possible for their deaths.

\section{Background}

Taiwan became an aging society in 1993. In 2018 adults aged 65 years and older represented $14.5 \%$ of the total population and it is estimated this will increase to $41.2 \%$ by $2065 .{ }^{11}$ In 2018, the number of deaths among people aged 65 years or older was 124,768 , which was an increase from the previous year and accounted for $72.2 \%$ of all deaths; the primary cause of death was cancer, followed by cardiac diseases and pneumonia. ${ }^{12}$ The quality of EoL of the elderly has gradually attracted attention.

Taiwan began promoting hospice care in the 1990s for patients who were considered to be terminally ill and in 2000 the first Hospice Palliative Care Act for self- determination of the terminally ill was established. ${ }^{13}$ Those in the terminal stage of a disease could subsequently receive palliative care instead of life-sustaining medical treatments such as a tracheotomy (TR) or CPR. Individuals are formally permitted to sign a living will for hospice palliative care, and family members are allowed to sign a do-not-resuscitate (DNR) request for relatives who are considered EoL. ${ }^{14}$

However, the first Hospice Palliative Care Act secured the right to make medical decisions only for patients who were terminally ill, which excluded patients who were critically ill, had a chronic disease, or were not yet considered "terminal". Therefore, on December 18, 2015, the Legislative Yuan, which is the unicameral legislature in Taiwan, passed the Patient Autonomy Act, which was formally implemented on January 6, 2019. This act proclaims that autonomous medical decisions are a patient's fundamental right and a universal human right. It states that, when the declarant is conscious, he or she may follow the advance care planning (ACP) procedure to complete the signing of ADs, autonomously deciding subsequent medical care choices subject to various clinical conditions and EoL stages. ${ }^{15}$

To date, for Asian older adults, signing ADs remains extremely difficult. Although ADs are based on personal autonomy, Chinese society emphasizes decisions that are made as a family or group and EOL care is profoundly influenced by family and group values. ${ }^{16}$ In addition, discussions about death have long been taboo in Eastern society. Unless absolutely necessary, the topic of death is rarely formally discussed among family members. For example, Lin et al reported that only $29.6 \%$ of inpatients had discussed EoL medical decisions with their families. ${ }^{17}$ A study by Matsumura et al found only $6 \%$ to $11 \%$ of older Japanese-American adults with chronic diseases had discussed EoL care with their physicians, ${ }^{18}$ demonstrating that this cultural taboo is maintained by immigrants in Western countries with Eastern cultural backgrounds.

Despite family members hope to make the best decision for their loved ones, the decisions made by family members may not necessarily meet the actual wishes of the elderly. ${ }^{19}$ In order to promote the decision-making of family members to be more consistent with the elderly's expectations for EoL treatments, more relevant studies are needed to explore the factors associated with the consistency of EoL medical treatment decision-making between the elderly and family members. 


\section{Patients and Methods \\ Research Design and Participants}

This was a cross-sectional and correlational study. Dyads consisting of older hospitalized patients and their primary family caregivers were recruited by convenience sampling from the internal medicine wards of a medical center in Central Taiwan. The primary family caregiver was the person nominated by the patient to oversee their care during their hospital stay. Inclusion criteria for patients were: (1) inpatient care provided by the department of internal medicine, (2) $\geq 65$ years of age, (3) ability to communicate in Chinese or Taiwanese, and (4) the patient's primary family caregiver also agreed to participate. Exclusion criteria were as follows: (1) the patient was $\geq 65$ years of age but had two or more incorrect answers to questions on the Short Portable Mental Status Questionnaire, ${ }^{20}$ (2) the patient's primary family caregiver was $<20$ years of age or had communication difficulties, and (3) the patient's primary caregiver was of a foreign nationality or a hired primary caregiver.

Estimation of sample size was determined with Software $G^{*}$ Power using the $\mathrm{z}$ test setting, ${ }^{21}$ with the odds ratio set to $1.68-1.73,{ }^{22} \alpha=0.05$, and the power $=$ 0.80 . The estimated sample size was 173-192 participants, equivalent to $87-96$ dyads.

\section{Measures}

\section{Demographic and Clinical Information}

Data were collected between February, 2017 and November, 2017. A general questionnaire collected demographic information for age, gender, ethnicity, marital status, and education of patients and caregivers. In addition, clinical information for patients included the presence or absence of cancer, and any comorbidities. Caregivers were asked what their relationship was to the patient. We asked patients and caregivers if they had previously been admitted to the intensive care unit (ICU), visited family or friends in the ICU, discussed EoL lifesustaining medical decisions with the caregiver participating the current study, signed a living will for hospice palliative care, or signed a DNR for other family members.

\section{Consistency}

Consistency was measured using the End-of-Life lifesustaining Medical Treatment-Preference Scale (EOLLSMT), which was developed by the research team and was based on the literature for EoL treatment decisions. ${ }^{5,8,22,23}$ Participants first read the following narrative describing an EOL scenario:

You have been diagnosed by a doctor as having an incurable disease and you are close to dying. It is difficult for you to breath, you are in severe pain, and your quality of life will be affected. You may even appear to be in a vegetative state. After reading about the possible life-sustaining medical treatments, which would you choose?

Consistency in this study was defined as the agreement between the preference of the hospitalized patient and what the caregiver inferred would be the patient's preference regarding life-sustaining medical treatments in an EoL scenario. Participants were given a description of seven life-sustaining medical treatments and the advantages and disadvantages of each treatment: cardiopulmonary resuscitation (CPR), intravenous (IV), nasogastric tube feeding (NG), admission to the intensive care unit (ICU), blood transfusion (BT), tracheotomy (TR), hemodialysis (HD). Patients were asked to respond to each life-sustaining medical treatment, based on the scenario, with "yes", "no", or "not sure". The primary family caregiver was provided with the same narrative and asked to infer the patient's response ("yes", "no", or "not sure"). The EOLLSMT scale was scored by comparing the responses between the patient and the caregiver. When the preference of the patient and the inference of the primary family caregiver were the same for an EoL life-sustaining medical treatment, a value of 1 was assigned; otherwise, a value of 0 was assigned. Consistency was recorded when the patient's answer matched the caregiver's inferred response.

\section{Mutuality Scale}

The mutuality scale was developed by Archbold et al to measure the positive qualities of relationships between primary family caregivers and older adults. ${ }^{24}$ It consists of 15 questions in four areas: love, shared pleasurable activities, shared values, and reciprocity. Each item is rated on a 5-point Likert scale, from 0 (almost none) to 4 (very much). The total score ranges from 0 to 60; a higher score indicates favorable mutuality. For the English version, the internal reliability yielded a Cronbach's $\alpha$ of $0.91 .{ }^{24}$ We used a Chinese version of the mutuality scale, which has been demonstrated to be a valid instrument for caregivers and older adults in Taiwan, with a Cronbach's $\alpha$ of $0.95 .{ }^{25}$ In this study, Cronbach's $\alpha$ was 0.95

\section{Modified Cumulative Illness Rating Scale-Geriatrics (CIRS-G)}

The modified CIRS-G scale provides quantitative ratings of chronic illness burden for common chronic medical problems affecting medically impaired persons 65 years of age and older. ${ }^{26}$ The CIRS-G is categorized according to 14 physiological systems including heart and lungs, visual, genitourinary, musculoskeletal, neurological, endocrine-metabolic, and 
psychiatric or behavioral illnesses. Each system is rated on a 5-point Likert-scale from 0 (no problem affecting the system) to 4 (extremely severe problem). The maximum total score is 56; a higher score indicates more comorbidities. The Chinese version of the CIRS-G has been widely used in major hospitals in Taiwan.

\section{Procedures}

This study was conducted in accordance with the Declaration of Helsinki and approved by the Institutional Review Board of Taichung Veterans General Hospital (No. CE16267B) prior to data collection. The first author explained the design and purpose of the study to both the hospitalized patients and their primary family caregivers. Only after all participants fully understood the procedures for this study and provided written consent did data collection begin.

\section{Data Collection}

Questionnaire interviews were conducted during each patient's hospital stay in the general internal medicine ward. The hospitalized patients and the primary family caregivers each received the questionnaires separately. First, we asked the primary family caregiver to go to a meeting room to rest, and we conducted one-on-one interviews with the patient in the ward regarding the questionnaires. The questionnaire interviews with the primary family caregivers were conducted in the meeting room.

\section{Statistical Methods}

SPSS software, version 22.0 (IBM SPSS Statistics) was used for data analysis. Descriptive statistics for variables included frequencies, percentages, means, and standard deviations (SD). Consistency between the views of hospitalized patients and their primary family caregivers on EoL life-sustaining medical treatment decisions are represented by percentages, the McNemar test was used to determine differences between consistencies, and the Kappa parameter test was used to determine the level of consistency between the two. The calculation results were between -1 and 1. Kappa values of $0-0.20,0.21-0.40,0.41-0.60,0.61-0.80$, and $0.81-1.00$ represent slight, fair, moderate, substantial, and almost perfect consistencies, respectively. ${ }^{27}$ Bivariate analysis (Chi-square test or $t$ test) was used to examine the correlation between the consistency of each independent variable and the seven life-sustaining medical treatments
(CPR, IV, NG, ICU admission, BT, TR, and HD). To determine if there were any predictors of consistency for hospitalized patients and family caregivers, we first performed a bivariate analysis to identify any independent variables that were correlated with preferences for the seven EoL life-sustaining medical treatments. Significantly correlated variables were then analyzed using binary logistic regression to establish the factors related to the consistency between the preferences of the hospitalized patient and primary family caregivers. A level of $\mathrm{p}<0.05$ was considered statistically significant.

\section{Results}

\section{Characteristics of the Dyads and Mutuality Between Patients and Their Family Caregivers}

A total of 220 patients met the inclusion criteria and 220 dyads were invited to participate; 48 patients were not interested in participating, and 72 family caregivers were opposed to the interview process. Therefore, 100 dyads of older hospitalized patients and their primary family caregivers were included in our study, for a total of 200 participants. Details of the characteristics of the dyads are shown in Table 1. Hospitalized patients had a mean age of 74.4 years $(\mathrm{SD}=7.43)$ and slightly more than half were male (54\%); most were married, of Hoklo Taiwanese ethnicity, and had an elementary school level of education or lower. The mean score on the CIRS-G was $10.9 \pm 6.43$ and $43 \%$ had been diagnosed with cancer. The mean age of the primary caregivers was 54.43 years $(\mathrm{SD}=12.7)$, and slightly more than half were female $(55 \%)$; most were children of the patient.

The number of personal medically related experiences differed between patients and family caregivers (Table 1): more than twice as many patients had been admitted to the ICU ( $45 \%$ of patients versus $21 \%$ of caregivers), and had signed a living will (18\% of patients versus $8 \%$ of caregivers); while twice as many caregivers had signed a DNR for another family member (13\% of caregivers versus $6 \%$ of patients). Although $68 \%$ of patients reported having discussed EoL life-sustaining medical treatment decisions with their family caregiver, only $43 \%$ of family caregivers reported having had this discussion with the patient. The mean scores on the Mutuality Scale were higher for hospitalized patients compared to caregivers (Table 1). 
Table I Characteristics of Dyads $(\mathrm{N}=100)$ of Older Hospitalized Patients and Their Primary Caregivers

\begin{tabular}{|c|c|c|c|c|}
\hline \multirow[t]{2}{*}{ Characteristics } & \multicolumn{2}{|c|}{ Patients } & \multicolumn{2}{|c|}{ Caregivers } \\
\hline & $\%$ & Mean (SD) & $\%$ & Mean (SD) \\
\hline Age (years) & & $74.40(7.43)$ & & $54.43(12.7)$ \\
\hline \multicolumn{5}{|l|}{ Gender } \\
\hline Male & 54 & & 45 & \\
\hline Female & 46 & & 55 & \\
\hline \multicolumn{5}{|l|}{ Ethnicity } \\
\hline Hoklo Taiwanese & 75 & & & 75 \\
\hline Other & 25 & & & 25 \\
\hline \multicolumn{5}{|l|}{ Marital status } \\
\hline Married & 70 & & & \\
\hline Other & 30 & & & \\
\hline \multicolumn{5}{|l|}{ Level of education } \\
\hline Elementary or lower & 74 & & & \\
\hline Junior high or lower & & & 29 & \\
\hline$\geq$ Junior high & 26 & & & \\
\hline$\geq$ High school & & & 71 & \\
\hline \multicolumn{5}{|l|}{ Clinical characteristics of patient } \\
\hline \multicolumn{5}{|l|}{ Diagnosis of cancer } \\
\hline Yes & 43 & & & \\
\hline No & 57 & & & \\
\hline CIRS-G score, $($ range $=2-40)$ & & $10.92(6.43)$ & & \\
\hline \multicolumn{5}{|l|}{ Relationship of caregiver to patient } \\
\hline Spouse & & & 32 & \\
\hline Child & & & 61 & \\
\hline Other & & & 7 & \\
\hline \multicolumn{5}{|l|}{ Personal medical-related experiences } \\
\hline Previously admitted to the ICU & 45 & & 21 & \\
\hline Visited friends or family in the ICU & 68 & & 83 & \\
\hline Discussed end-of-life medical decisions ${ }^{\mathrm{a}}$ & 68 & & 43 & \\
\hline Signed a living will & 18 & & 8 & \\
\hline Signed a DNR for other family members & 6 & & 13 & \\
\hline Mutuality scale score & & 43.12 (11.39) & & $38.98(12.08)$ \\
\hline
\end{tabular}

Note: ${ }^{a}$ Discussed end-of-life medical decisions means patient and primary caregiver have talked about these decisions with one another. Abbreviation: CIRS-G, Modified Cumulative Illness Rating Scale-Geriatrics (maximum score = 56).

\section{Consistency of EoL Life-Sustaining Medical Treatment Preferences Between Hospitalized Patients and Caregivers}

When preferences for EOL life-sustaining medical treatments were compared, more hospitalized patients chose "no" for all treatments (71-88\%) than primary caregivers. Most primary caregivers chose "no" only for CPR, NG feeding, and TR, and "not sure" for all other treatments. For all treatments, the proportion of primary family caregivers choosing "yes" was higher than the proportion of patients for the corresponding treatment. Consistencies between preferences of EoL lifesustaining treatments for hospitalized patients and primary family caregivers are shown in Table 2. Consistencies were highest for tracheotomy $(60 \%)$ and lowest for BT $(33 \%)$. Across all treatments, consistency averaged $42.3 \%$ and was highest for the choice of "no", which ranged from $21 \%$ for BT to $52 \%$ for TR. McNemar tests showed the differences in consistencies for all treatment preferences were statistically significant $(\mathrm{p}<0.001)$. However, the Kappa test showed the only consistencies that significantly differed between groups 
Table 2 Consistency Between Decisions for Hospitalized Patients and Their Primary Caregivers Regarding EOL Life-Sustaining Medical Treatments

\begin{tabular}{|c|c|c|c|c|c|c|c|}
\hline Medical Treatment & $\begin{array}{l}\text { Patients } \\
\text { (\%) }\end{array}$ & $\begin{array}{l}\text { Caregivers } \\
\text { (\%) }\end{array}$ & $\begin{array}{l}\text { Consistency } \\
\text { (\%) }\end{array}$ & McNemar Test & p value & Kappa $(95 \%$ Cl) & p value \\
\hline $\begin{array}{l}\text { Cardiopulmonary resuscitation } \\
\text { Yes } \\
\text { No } \\
\text { Not sure }\end{array}$ & $\begin{array}{l}3 \\
88 \\
9\end{array}$ & $\begin{array}{l}16 \\
48 \\
36\end{array}$ & $\begin{array}{l}46 \\
0 \\
43 \\
3\end{array}$ & 33.857 & $<0.001$ & $0.001(0.970,1.000)$ & $>0.999$ \\
\hline $\begin{array}{l}\text { Intravenous therapy } \\
\text { Yes } \\
\text { No } \\
\text { Not sure }\end{array}$ & $\begin{array}{l}13 \\
71 \\
16\end{array}$ & $\begin{array}{l}24 \\
33 \\
43\end{array}$ & $\begin{array}{l}35 \\
2 \\
24 \\
9\end{array}$ & 27.236 & $<0.001$ & $0.024(0.546,0.734)$ & 0.640 \\
\hline $\begin{array}{l}\text { Nasogastric feeding tube } \\
\text { Yes } \\
\text { No } \\
\text { Not sure }\end{array}$ & $\begin{array}{l}4 \\
84 \\
12\end{array}$ & $\begin{array}{l}18 \\
46 \\
36\end{array}$ & $\begin{array}{l}48 \\
0 \\
41 \\
7\end{array}$ & 30.089 & $<0.001$ & $0.077(0.113,0.267)$ & 0.190 \\
\hline $\begin{array}{l}\text { Intensive care unit } \\
\text { Yes } \\
\text { No } \\
\text { Not sure }\end{array}$ & $\begin{array}{l}7 \\
76 \\
17\end{array}$ & $\begin{array}{l}33 \\
28 \\
39\end{array}$ & $\begin{array}{l}33 \\
1 \\
23 \\
9\end{array}$ & 45.922 & $<0.001$ & $0.040(0.442,0.638)$ & 0.540 \\
\hline $\begin{array}{l}\text { Blood transfusion } \\
\text { Yes } \\
\text { No } \\
\text { Not sure }\end{array}$ & $\begin{array}{l}9 \\
74 \\
17\end{array}$ & $\begin{array}{l}35 \\
25 \\
40\end{array}$ & $\begin{array}{l}33 \\
5 \\
21 \\
7\end{array}$ & 45.752 & $<0.001$ & $0.064(0.174,0.346)$ & 0.260 \\
\hline $\begin{array}{l}\text { Tracheotomy } \\
\text { Yes } \\
\text { No } \\
\text { Not sure }\end{array}$ & $\begin{array}{l}1 \\
87 \\
12\end{array}$ & $\begin{array}{l}12 \\
56 \\
32\end{array}$ & $\begin{array}{l}60 \\
0 \\
52 \\
8\end{array}$ & 25.667 & $<0.001$ & $0.155(0.000,0.030)$ & 0.010 \\
\hline $\begin{array}{l}\text { Hemodialysis } \\
\text { Yes } \\
\text { No } \\
\text { Not sure }\end{array}$ & $\begin{array}{l}6 \\
82 \\
12\end{array}$ & $\begin{array}{l}22 \\
34 \\
44\end{array}$ & $\begin{array}{l}41 \\
2 \\
31 \\
8\end{array}$ & 43.156 & $<0.001$ & $0.100(0.002,0.078)$ & 0.40 \\
\hline
\end{tabular}

Abbreviation: $\mathrm{Cl}$, confidence interval.

were for treatment with TR and HD (Kappa $=0.155$ and 0.100 , respectively, $\mathrm{p}<0.05$ ) (Table 2).

\section{Predictors of Consistency Between Preferences for EoL Life-Sustaining Treatments for Hospitalized Patients and Primary Family Caregivers}

All correlated significant variables examined in the logistic regression model, using stepwise analysis, are shown in Table 3. Significant predictors of consistency in providing CPR were a patient who had signed a living will (odds ratio $[\mathrm{OR}]=6.20, \mathrm{p}=<0.01$ ). In contrast, the gender of the caregiver (male) was not only a negative predictor of consistency in providing $\mathrm{CPR}(\mathrm{OR}=0.23, \mathrm{p}=<0.01)$ but also for providing IV treatment $(\mathrm{OR}=0.42, \mathrm{p}<0.05)$. There were two predictors of consistency for initiating an NG feeding: a patient who had visited friends or relatives in the ICU $(\mathrm{OR}=2.94, \mathrm{p}<0.05)$ and a caregiver who was the spouse of the patient $(\mathrm{OR}=3.07, \mathrm{p}<0.05)$. Consistency for BT was predicted by the marital status of the patient $(\mathrm{OR}=3.33, \mathrm{p}<0.05)$. Caregivers who had visited friends in the ICU and were the spouse of the patient were predictors of consistency for $\mathrm{TR}(\mathrm{OR}=5.50$, $\mathrm{p}<0.01$; and $\mathrm{OR}=3.12, \mathrm{p}<0.05$, respectively) and HD $(\mathrm{OR}=4.42, \mathrm{p}<0.05$ and $\mathrm{OR}=2.76, \mathrm{p}<0.05$, respectively). The CIRS-G score for hospitalized patients was the only independent variable significantly correlated with 


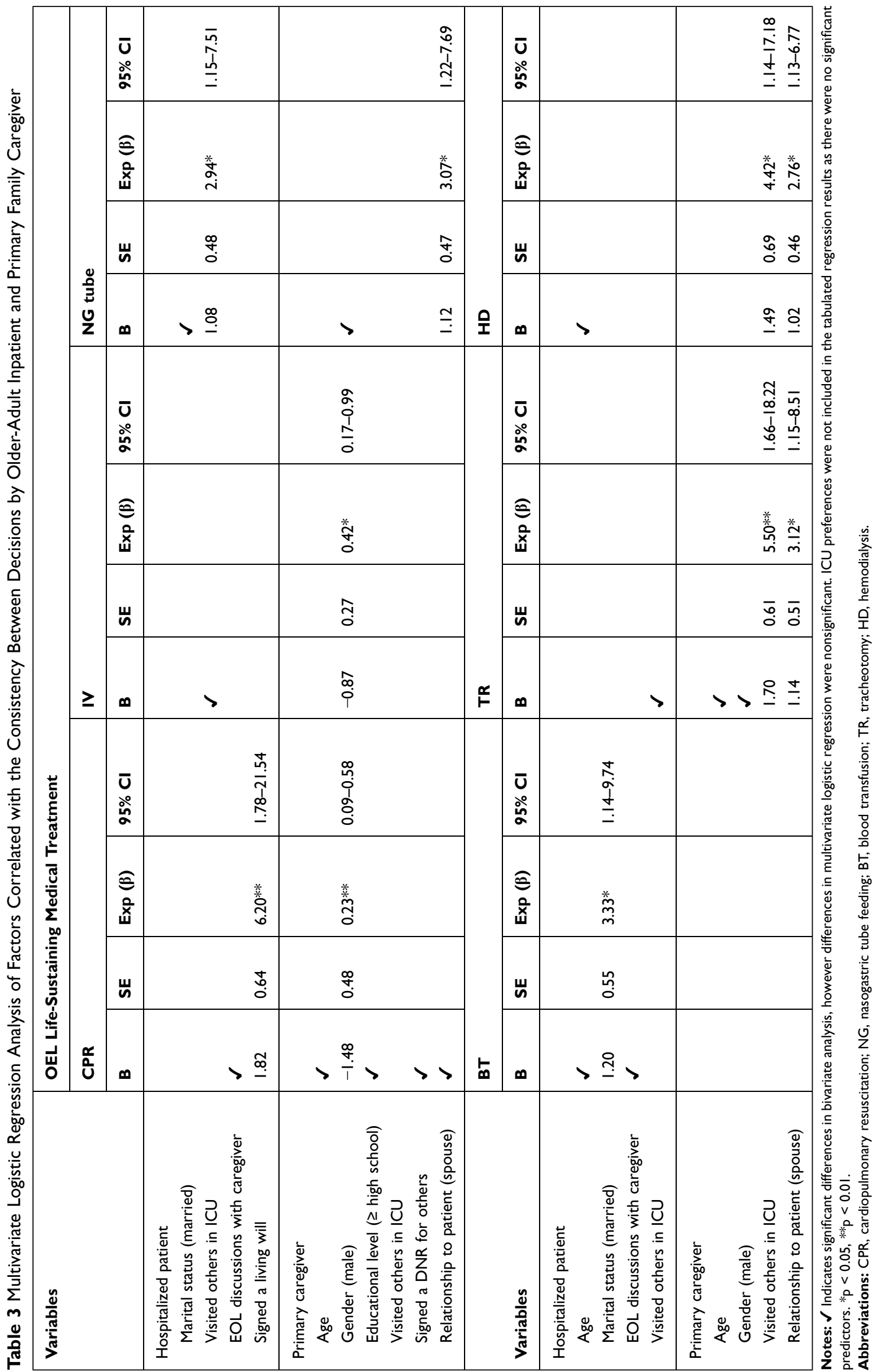


treatment in the ICU; however, it was not a significant predictor in the regression model.

\section{Discussion}

Our finding that family members overestimated the preferences of the patients, is consistent with previous studies. ${ }^{8,19}$ Family members have been shown to view lifesustaining medical treatments as proactive and believe terminating treatment is the premature termination of the life of the older adult, ${ }^{19}$ or have higher expectations regarding the potential use of life-sustaining medical treatments than the patient themselves. ${ }^{8}$

Family members also believed the use or withdrawal of life-sustaining medical treatments was equivalent to choosing life or death for the patient. This may be similar to situations where family members are more likely to choose life-sustaining medical treatments rather than adhere to the wishes of the patient for fear of being accused of depriving the patient of life or of shunning their caregiving responsibilities ${ }^{28,29}$ in contrast to preferences of older adults who have no misgivings about accepting EoL events as a natural occurrence. ${ }^{29}$ Therefore, the decisions of the primary family caregivers in our study may reflect their personal expectations regarding the circumstances of the patient or social expectations rather than the wishes of the hospitalized patient. $^{30}$

Consistency implied patients' wishes regarding EoL care could be understood and implemented. However, the average consistency was $42 \%$, which is lower than reported in previous studies $(60.5 \%-68.0 \%)^{5,7,8,22,23}$ The low consistency may be due to the design of the questionnaire, which included the response "not sure" as an option in situations in which the patient and caregivers had not discussed issues surrounding EoL care. Consistency between the patient's and caregiver's preferences was low for family caregivers of older adults with early dementia diagnosed with cancer who had not engaged in relevant discussions with the patient. ${ }^{6}$ However, the low consistency might also be due to patients' preferences having been unclear, or they felt uncertain about their preferences, even after discussions. In addition, social pressure may have prevented caregivers from answering "no" and the "not sure" option was less distressing. It has been suggested that discussions with caregivers about the decision-making process for EOL care should include assessments of decisional conflicts, such as social pressure, and to facilitate communication between the caregiver, patient, and medical personnel about these conflicts. ${ }^{31-33}$

Prior discussions about EoL medical treatments were not associated with consistency regarding preferences, which is in contrast to the findings of Barrio-Cantelejo et al. ${ }^{5}$ This difference might be explained by the fact that patients and caregivers had different perceptions of having had informal discussions about EoL medical treatments; $68 \%$ of hospitalized patients reported having discussions with their caregivers, whereas only $48 \%$ of caregivers reported having discussed the topic with the patients. It is also possible that the patients had discussed EoL care preferences with other family members, but the primary caregivers were not aware of the patients' discussion.

Two additional differences, which involve discussions about EoL care, might also explain the low level of consistency in our study. First, although $43 \%$ of the patients had been diagnosed with cancer, none were in a terminal stage. Patients with terminal cancer and their family members are more likely to have had opportunities to consider and discuss their expectations regarding EoL care. ${ }^{8,22}$ Second, discussions about death are taboo in Chinese cultures that are influenced by Confucianism, such as Taiwan. Older adults rarely take a proactive approach for EoL medical care, such as providing instructions to their families. There is a lack of discussion between patients, primary family caregivers, and medical staff in Chinese cultures, which results in a greater discrepancy between family members' expectations regarding EoL life-sustaining medical care than for Western cultures. ${ }^{17}$

The highest level of consistency was a lack of interest in treatment involving a tracheotomy $(60 \%)$, which may be influenced by culture. Tracheotomy involves an obvious effect on physical appearance and signifies pain, incurability, and a long-term illness. $^{34}$ Consistency between patients and caregivers for treatment with NG feeding, TR, and HD was positively correlated with a primary family caregiver who was the spouse of the patient. This finding corresponds with that of Parks et al, ${ }^{35}$ who demonstrated consistency in elder-proxy relationships for life-sustaining medical decisions was highest when the proxy was a spouse. Although Foo et al found that when faced with EoL life-sustaining medical decisions, older adults preferred to let their adult children rather than their spouse make decisions. ${ }^{23}$ However, Parks et al demonstrated the lowest consistency in elder-proxy 
relationships occurred when the proxy was an adult child. ${ }^{35}$ This may explain why a family caregiver who was a child of the hospitalized patient was not a predictor for any of the life-sustaining medical treatments.

Male family caregivers were negatively correlated with consistency in preferences for CPR and IV treatments. This finding may because female caregivers spend more time providing care and have more opportunities to observe and understand the patient's thoughts. ${ }^{36}$ This difference might also be explained by the cultural taboo of discussing death. Male family caregivers may be less likely to have discussions about EoL care with the hospitalized patient, and therefore lack the information necessary to accurately predict the wishes of the patient.

The signing of a living will for hospice palliative care by hospitalized patients was a significant predictor of consistency for CPR. An intervention study in Spain showed there was a greater consistency for EoL lifesustaining medical decisions between older patients and their assigned proxies when the patient had signed an $\mathrm{AD}$ compared with those who had not; and consistency was even greater between patients who had discussed ACP with their proxies regarding life-sustaining medical preferences. ${ }^{5}$ Effective communication that allows the patient and their primary caregiver time to consider and discuss topics regarding EoL medical treatment and care has been shown to increase consistency in EoL medical care preferences. ${ }^{37}$ In addition, ACP can help older adults understand the importance of ADs. ${ }^{38}$

Previous visits to friends or relatives in the ICU were predictors of consistency for treatments, but treatments differed between patients and caregivers. A hospitalized patient who had visited the ICU was a predictor of NG feeding, while a caregiver who had visited the ICU was a predictor for TR and HD. Foo et al showed that older adults with a friend or relative in the ICU exhibited greater consistency with their proxies, which can influence one's perspective on whether to undergo life support treatment. ${ }^{23}$ Tracheotomy, NG feeding, and hemodialysis are common medical procedures in the ICU. Therefore, having observed someone of importance in the ICU may have provided patients and caregivers with a real-life experience of what is involved in critical care and indirectly increased consistency between patients and caregivers in our study. A lack of personal experience or observation of procedures in the ICU can make it difficult to understand life-sustaining medical treatments in the absence of professional guidance.

Previous studies have demonstrated that the quality and intimacy of relationships between older adults and family members are correlated with EoL life-sustaining medical decisions. ${ }^{35,39}$ However, in the present study, mutuality was not significantly correlated with consistency in EoL treatment preferences. This may be another variable that differs from other studies due to the avoidance of discussions surrounding death by Chinese families. Therefore, mutuality between patients and primary family caregivers did not increase consistency between these two parties regarding preferences for EoL life-sustaining medical treatments.

\section{Limitations}

This study had several limitations and, therefore, the findings should be interpreted with caution. The crosssectional design of the study does not allow us to draw conclusion about causality with respect to preferences for EoL treatments. We recruited hospitalized patients from the internal medicine wards of one medical center in Taiwan, which might limit the generalization of our findings to other areas of Taiwan and other hospitalized patients with different critical care needs. In addition, the use of convenience sampling has the potential for selection bias, given the high refusal rate of patients and caregivers during recruitment. Decisions about EoL remain a sensitive issue in Taiwanese culture and often involve complex family dynamics and history; however, we did not collect information about the role or legal power of the primary caregiver in these decisions. Finally, caution must be taken in interpreting these findings because the self-report information surrounding the scenario may not reflect the actual preferences of hospitalized patients for EoL treatments.

\section{Conclusion}

This study examined the consistency of preferences between older hospitalized patients and primary family caregivers by asking caregivers to infer what the preferences would be for the patients with regard to seven EoL life-sustaining medical treatments. When participants were presented with the scenario, which described a hospitalized patient close to dying, most family caregivers inferred the patient would choose "not sure" for four of the seven EoL medical treatments, whereas most patients selected "no" for all treatments. The consistency 
was low for preferences of end-of-life medical care between patients and caregivers. Facilitating communication about ACP through discussions, patients and family member can clarify their expectations regarding EoL medical care, values, beliefs, quality of life, and treatment preferences, thereby promoting consistency in EoL care decisions of older patients and their families.

\section{Recommendations}

To increase consistency in EoL life-sustaining medical preferences for older hospitalized patients and their primary family caregivers, we provide the following recommendations based on our study findings: (1) Both children and spouses should participate in discussing EoL medical care, and should be encouraged to discuss personal experiences of their friends' or family members' admission to hospitals or ICUs; (2) To be policy-wise, medical institutes should provide frequent ACP counseling, enabling family members and older adults' to discuss the positive and negative outcomes of EoL medical treatments; (3) Medical staffs should understand the cultural values and decision conflict of older hospitalized patients and their caregivers when developing ACP.

\section{Acknowledgments}

This research was supported by the Ministry of Science and Technology (MOST 106-2314-B-182 -006 -MY3 and MOST 109-2314-B-227 -006 -MY3) and Teaching Practice Research Program of Ministry of Education (PMN1080130). We would like to thank all the elderly patients and their primary family caregivers who took part in the study.

\section{Disclosure}

All authors report no conflicts of interest in this work.

\section{References}

1. Marco CA, Bessman ES, Kelen GD. Ethical issues of cardiopulmonary resuscitation: comparison of emergency physician practices from 1995 to 2007. Acad Emerg Med. 2009;16(3):270-273. doi:10.1111/j.15532712.2008.00348.x

2. Fritz Z, Slowther A-M, Perkins GD. Resuscitation policy should focus on the patient, not the decision. $B M J .2017 ; 356: \mathrm{j} 813$. doi:10.1136/bmj. j813

3. Bunn F, Goodman C, Russell B, et al. Supporting shared decision making for older people with multiple health and social care needs: a realist synthesis. BMC Geriatrics. 2018;18(1):165. doi:10.1186/ s12877-018-0853-9

4. Huang H-L, Shyu Y-IL, Weng L-C, Chen K-H, Hsu W-C. Predictors of advance directives among nursing home residents with dementia. Int Psychogeriatr. 2018;30(3):341-353. doi:10.1017/S10416102 17001661
5. Barrio-Cantalejo IM, Molina-Ruiz A, Simón-Lorda P, et al. Advance Directives and Proxies' Predictions About Patients' Treatment Preferences. Nursing Ethics. 2009;16(1):93-109. doi:10.1177/ 0969733008097995

6. Harrison Dening K, King M, Jones L, Vickestaff V, Sampson EL, Paci E. Advance care planning in dementia: do family carers know the treatment preferences of people with early dementia? PLoS One. 2016;11(7):e0159056. doi:10.1371/journal.pone.0159056

7. Shalowitz DI, Garrett-Mayer E, Wendler D. The accuracy of surrogate decision makers: a systematic review. Arch Intern Med. 2006;166(5):493-497. doi:10.1001/archinte.166.5.493

8. Tang ST, Liu T-W, Lai M-S, Liu L-N, Chen C-H. Concordance of preferences for end-of-life care between terminally ill cancer patients and their family caregivers in Taiwan. J Pain Symptom Manage. 2005;30(6):510-518. doi:10.1016/j.jpainsymman.2005.05. 019

9. Ho ZJM, Radha Krishna LK, Yee CPA. Chinese familial tradition and western influence: a case study in Singapore on decision making at the end of life. J Pain Symptom Manage. 2010;40(6):932-937. doi:10.1016/j.jpainsymman.2010.06.010

10. Yarnell CJ, Fu L, Bonares MJ, Nayfeh A, Fowler RA. Association between Chinese or South Asian ethnicity and end-of-life care in Ontario, Canada. CMAJ. 2020;192(11):E266-E274. doi:10.1503/ cmaj. 190655

11. National Development Council, Taiwan, ROC. Population projections for Taiwan (2018 2065); 2018. Available from: https://www. ndc.gov.tw/Content_List.aspx?n=84223C65B6F94D72. Accessed September 26, 2018.

12. Ministry of Health and Welfare, Taiwan, ROC. Analysis of cause of death statistics; 2016. Available from: http://www.mohw.gov.tw/dl33660-b7dfc331-94bb-4da3-ae09-6aa58a3ee861.html. Accessed June 26, 2018.

13. Ministry of Health and Welfare, Taiwan, ROC. Hospice-Palliative Care Act; 2016. Available from: http://www.nhi.gov.tw/webdata/web data.aspx?menu $=20 \&$ menu_id $=712 \&$ webdata_id $=3651$. Accessed September 10, 2019.

14. Hospice Foundation of Taiwan, Taiwan, ROC. Hospice and Palliative Care/The Patient Autonomy Act; 2019. Available from: https://www. hospice.org.tw/care. Accessed September 10, 2019.

15. Ministry of Health and Welfare, Taiwan, ROC. The Patient Autonomy Act will be explained next year, and the Department of Health and Welfare announces the complementary measure; 2019. Available from: https://www.mohw.gov.tw/cp16-44221-1.html. Accessed June 26, 2019.

16. Jo K-H, An G-J, Han KS. Family factors affecting on withdrawal of life-sustaining treatment in Korea. International Journal of Nursing Practice. 2012;18(6):552-558. doi:10.1111/ijn.12009

17. Lin HM, Yang CL, Chen MM, Chiu TY, Hu WY. Inpatients' willingness on and acceptance of promotion for signing of advance directives. Taiwan J Hosp Palliat Care. 2011;16(3):281-295. doi:10.6537/TJHPC.2011.16(3).1

18. Matsumura S, Bito S, Liu H, et al. Acculturation of attitudes toward end-of-life care. $J$ Gen Intern Med. 2002;17(7):531-539. doi:10.1046/j.1525-1497.2002.10734.x

19. Suen L-JW, Lee $-\mathrm{H}-\mathrm{H}$, Morris DL. Life-sustaining treatment: a comparison of the preferences of Taiwanese older adults and their family caregiver. Journal of Nursing Research. 2013;21(4):261-269. doi:10.1097/JNR.000000000000006

20. Pfeiffer E. A Short Portable Mental Status Questionnaire for the Assessment of Organic Brain Deficit in Elderly Patients†. $J \mathrm{Am}$ Geriatr Soc. 1975;23(10):433-441. doi:10.1111/j.1532-5415.1975. tb00927.x

21. Faul F, Erdfelder E, Lang A-G, Buchner A. G*Power 3: A flexible statistical power analysis program for the social, behavioral, and biomedical sciences. Behav Res Methods. 2007;39(2):175-191. doi:10.3758/BF03193146 
22. Tang ST, Chen CC-H, Tang W-R, Liu T-W. Determinants of PatientFamily Caregiver Congruence on Preferred Place of Death in Taiwan. J Pain Symptom Manage. 2010;40(2):235-245. doi:10.1016/j. jpainsymman.2009.12.018

23. Foo AS, Lee TW, Soh CR. Discrepancies in end-of-life decisions between elderly patients and their named surrogates. Ann Acad Med Singap. 2012;41(4):141-153.

24. Archbold PG, Stewart BJ, Greenlick MR, Harvath T. Mutuality and preparedness as predictors of caregiver role strain. Research in Nursing \& Health. 1990;13(6):375-384. doi:10.1002/ nur.4770130605

25. Huang H-L, Weng L-C, Tsai Y-H, et al. Predictors of self- and caregiver-rated quality of life for people with dementia living in the community and in nursing homes in northern Taiwan. Int Psychogeriatr. 2015;27(5):825-836. doi:10.1017/ s1041610214002506

26. Miller MD, Paradis CF, Houck PR, et al. Rating chronic medical illness burden in geropsychiatric practice and research: application of the Cumulative Illness Rating Scale. Psychiatry Res. 1992;41 (3):237-248. doi:10.1016/0165-1781(92)90005-N

27. Landis JR, Koch GG. The Measurement of observer agreement for categorical data. Biometrics. 1977;33(1):159-174. doi:10.2307/ 2529310

28. Schenker Y, Crowley-Matoka M, Dohan D, Tiver GA, Arnold RM, White DB. I don't want to be the one saying 'we should just let him die': intrapersonal tensions experienced by surrogate decision makers in the ICU. J Gen Intern Med. 2012;27(12):1657-1665. doi:10.1007/ s11606-012-2129-y

29. Tang CSH, Lam LCW, Chiu HFK. Attitudes to end-of-life decisions: a survey of elderly Chinese with dementia and their carers. Asian $J$ Gerontol Geriatr. 2007;2:119-125.

30. Marks MAZ, Arkes HR. Patient and Surrogate Disagreement in Endof-Life Decisions: can Surrogates Accurately Predict Patients Preferences? Med Decis Making. 2008;28(4):524-531. doi:10.1177/ 0272989X08315244
31. Rafferty KA, Cramer EM, Priddis D. Managing end-of-life uncertainty: applying problematic integration theory to spousal communication about death and dying. Am J Hosp Palliat Care. 2016;33 (1):69-76. doi:10.1177/1049909114550675

32. Ridley S, Fisher M. Uncertainty in end-of-life care. Curr Opin Crit Care. 2013;19(6):642-647. doi:10.1097/mcc.000000000000003

33. Huang H-L, Lu W-R, Liu C-L, Chang H-J, Ginsberg SD. Advance care planning information intervention for persons with mild dementia and their family caregivers: impact on end-of-life care decision conflicts. PLoS One. 2020;15(10):e0240684. doi:10.1371/journal. pone. 0240684

34. Chen HC, Wang HH. The management and utilization of tracheostomy care for ventilator-dependent patients - A literature review. Chang Gung J Sci. 2014;21:25-38. doi:10.6192/ CGUST.2014.12.21.3

35. Parks SM, Winter L, Santana AJ, et al. Family factors in end-of-life decision-making: family conflict and proxy relationship. J Palliat Med. 2011;14(2):179-184. doi:10.1089/jpm.2010.0353

36. Sharma N, Chakrabarti S, Grover S. Gender differences in caregiving among family - caregivers of people with mental illnesses. World J Psychiatry. 2016;6(1):7-17. doi:10.5498/wjp.v6.i1.7

37. El-Jawahri A, Traeger L, Shin JA, et al. Qualitative Study of Patients and Caregivers" Perceptions and Information Preferences About Hospice. J Palliat Med. 2017;20(7):759-766. doi:10.1089/ jpm.2016.0104.

38. Nicholson C, Morrow EM, Hicks A, Fitzpatrick J. Supportive care for older people with frailty in hospital: an integrative review. Int J Nurs Stud. 2017;66:60-71. doi:10.1016/j.ijnurstu.2016.11.015

39. Turan B, Goldstein MK, Garber AM, Carstensen LL. Knowing loved ones' end-of-life health care wishes: attachment security predicts caregivers“ accuracy. Health Psychol. 2011;30(6):814-818. doi: $10.1037 / \mathrm{a} 0025664$
Patient Preference and Adherence

\section{Publish your work in this journal}

Patient Preference and Adherence is an international, peer-reviewed, open access journal that focusing on the growing importance of patient preference and adherence throughout the therapeutic continuum. Patient satisfaction, acceptability, quality of life, compliance, persistence and their role in developing new therapeutic modalities and compounds to optimize clinical outcomes for existing disease states are major areas of interest for the journal. This journal has been accepted for indexing on PubMed Central. The manuscript management system is completely online and includes a very quick and fair peer-review system, which is all easy to use. Visit http:/ www.dovepress.com/testimonials.php to read real quotes from published authors. 